\title{
Nonlinear Electrical Conductivity Properties of Au Films Prepared by Sputtering
}

\author{
Qingyun Meng, ${ }^{1}$ Yixin Kang, ${ }^{1}$ Xiaoyu Zhai, ${ }^{1}$ Ziwen Yin, ${ }^{1}$ and Dongpeng Yan $^{1,2}$ \\ ${ }^{1}$ State Key Laboratory of Chemical Resource Engineering, Beijing University of Chemical Technology, Beijing 100029, China \\ ${ }^{2}$ Key Laboratory of Theoretical and Computational Photochemistry, Ministry of Education, College of Chemistry, \\ Beijing Normal University, Beijing 100875, China
}

Correspondence should be addressed to Qingyun Meng; mqybuct@163.com, Ziwen Yin; yinziwen1989@163.com and Dongpeng Yan; yandp@mail.buct.edu.cn

Received 10 March 2014; Accepted 7 July 2014; Published 13 August 2014

Academic Editor: Ugur Serincan

Copyright ( 2014 Qingyun Meng et al. This is an open access article distributed under the Creative Commons Attribution License, which permits unrestricted use, distribution, and reproduction in any medium, provided the original work is properly cited.

\begin{abstract}
Metal-based films with tunable electrical conductivity have played an important role in developing new types of electric devices for future application. In this work, a sputtering method was used to obtain Au films on silicon substrate in a hypobaric atmosphere. Scanning electron microscope (SEM) shows that the interspaces between the Au nanoparticles were highly uniform and orderly distributed, with the width of several nanometers at the surface. By measuring the $I-V$ curves of the films with thickness less than $20 \mathrm{~nm}$, the nonlinear behaviors of electrical resistivity became gradually obvious as the decrease of the film thickness. For example, upon the thickness reducing to $10 \mathrm{~nm}$, remarkable discontinuous step phenomenon appeared. Moreover, a computational simulation was carried on the electrical conductivity of films under normal temperature based on the Coulomb blockade theory and scattering theory, in which the electric current was in the range from 0 to $1.5 \times 10^{-5} \mathrm{~A}$. The computational results were consistent well with the experimental observations, which confirm that the nonlinear and step phenomenon can be assigned to the Coulomb blockade effect when electrons transfer occurs in the interspaces between the nanoparticles.
\end{abstract}

\section{Introduction}

In recent years, due to the rapid development of electric and electronic devices, extensive study has been focused on the interface behaviors of metal-metal and metal-semiconductor. Under low-temperature condition (such as $77 \mathrm{~K}$ ), it was found that there was Coulomb blockade phenomenon in nanocrystalline metal with island structures $[1,2]$. When the thickness of metal film was at the nanometer scale, the structures became discontinuous [3], which were composed of isolated nanoparticles; while the thickness of film was close to De Broglie wavelength, the energy level became in discrete state, and the motion of electrons was perpendicular to the surface of films, which presented quantum size effect $[4,5]$. In the latter case, the resistivity of film was very large, and the temperature coefficient of resistance (TCR) usually became a minus value, which presented Ohms feature at low electric field but presented non-Ohms feature $[6,7]$ at high electric field. A large number of experimental reports show the jumping transmission of current carriers in interspace. That is, if the particle size in the nanoscaled film is small enough, electron transfer will be restrained, and the resistivity of films will show a jump with the increasing of voltage. However, up to date, there was no report about the Coulomb blockade phenomenon at room temperature, which has restricted such effect into the practical applications. Coulomb blockade phenomenon and macroscopic quantum tunneling phenomenon will play a significant role in the research and production of single electron device $[8,9]$, which will improve the degree of integration of integrated circuit. In this work, the nonlinear electrical conductivity of the nanostructured $\mathrm{Au}$ films was studied at room temperature. We further performed theoretical calculation, which agreed well with experimental results. By the combination of experimental and computational study on the discontinuous electrical conductivity of films, this work may pave an alternative way of metal films for further device application. 


\section{Experiments}

2.1. Experimental Materials and Instrument. Single-sidepolished silicon wafer was purchased from Tianjin Zhaoyi Jinke Technology Co., Ltd; Au sputtering target (purity: 99.99\%) was purchased from Beijing Zhongke Jinyu Technology Co., Ltd; HF solution (40\% or higher) was purchased from Beijing Chemical Works; anhydrous ethanol solution (mass fraction: $99.7 \%$ or higher) was purchased from Beijing Chemical Works; graphite electrode was purchased from Beijing Jixing Sheng'an Industry \& Trade Co., Ltd.

The Au nanoparticles were prepared on a DH2010 type vacuum evaporation coating machine (Hangzhou Dahua Instrument Co., Ltd) and a JZ-BJJY-type 2 DC sputtering apparatus (Beijing Zhongke Jinyu Technology Co., Ltd). Surface morphology was observed on an S-4700 type ice emission scanning electron microscope (SEM, HITASCHI). The electric measurement was performed on a TR-KDYtype 1 four-point probe resistivity meter (Beijing Tong De Technology Co., Ltd). The $I-V$ curve was measured on an HP4145 type semiconductor parameter analyzer and 122 type nanoampere table (Agilent companies in the United States). The thickness of film was estimated on an SE200BA-M300 type ellipsometer (American Stress Technologies, Inc.). The electrical conductivity of Au films with different thicknesses was measured at room temperature by the nanoampere table in the range from $\pm 2 \times 10^{-3} \mathrm{~A}$ to $\pm 1 \times 10^{-15} \mathrm{~A}$, and the elementary accuracy was $0.5 \%$ to $1.0 \%$.

2.2. Preparation for Au Nanometer Films. The electrolytic cell anode corrosion device was used to corrode the silicon wafer $(4 \mathrm{~cm} \times 0.8 \mathrm{~cm})$. The total volume of solution was $200 \mathrm{ml}$, containing HF solution and anhydrous ethanol solution, and the mixing proportion (bulk factor) was $1.8: 1$. The corrosion current was $45 \mathrm{~mA}$, and the corrosion time was 45 minutes. Corroded silicon wafer was put into LPCVD to achieve the growth of $\mathrm{SiO}_{2}$ films with pressure of 760 torr, temperature of $650^{\circ} \mathrm{C}$, and the oxidation time of 30 minutes in $99.99 \%$ pure oxygen environment. Finally, low vacuum DC sputtering method was employed to grow Au nanometer films under 5 $\times 10^{-2} \mathrm{~Pa}$. Multiple measurements were performed to confirm the results.

\section{Results and Discussion}

3.1. Volt-Ampere Characteristics of Au Films with Different Thickness. Figure 1(a) shows the typical I-V curve of Au film with the thickness of $20 \mathrm{~nm}$, in which the slope of the $I-V$ curve shows gradual change characteristic, and the threshold voltage is located at $1.8 \mathrm{~V}$, which presents the $I-V$ behaviors of typical semiconductors. Figure $1(\mathrm{~b})$ shows the $I-V$ curve of Au film with the thickness of $13 \mathrm{~nm}$, and there are several peaks of the current in the range from 6 to $9.1 \mathrm{~V}$. Therefore, the $I-V$ curve of Au nanometer films presents discrete electrical conductivity. Figure 1(c) is the $I-V$ curve of Au film with the thickness of $9 \mathrm{~nm}$, and it is found that the curve possesses steps structure, and the threshold voltage is $7.5 \mathrm{~V}$. There is no step until the voltage increases to $7.5 \mathrm{~V}$, and there is only one peak at the voltage of $4 \mathrm{~V}$. Figure $1(\mathrm{~d})$ is the $I-V$ curve of $\mathrm{Au}$ thin film with the thickness of $4 \mathrm{~nm}$. There is a jump when the voltage is $0.5 \mathrm{~V}$, and the steps appear in succession with the increasing of voltage. These results also show that the current values $(I)$ of the films are in the range of the order of magnitude of microampere.

3.2. Surface Morphology of Au Nanometer Films. Figure 2 shows the FE-SEM micrographs of Au nanometer films with the thickness of 13,9 , and $4 \mathrm{~nm}$, from which it can be observed that the as-prepared two-dimensional metal thin film is highly uniform, which is composed of discontinuous metal particle clusters. There are interspaces whose separation distance is in the range from 1 to $5 \mathrm{~nm}$ between the particles on the surface of the samples, and such an interspace may give rise to the discontinuous current change upon the increasing voltage.

3.3. Theoretical Calculation of the Electrical Conductivity of Films. According to FE-SEM micrographs of films in Figure 2, it is supposed that there are metal island capacitors at the surface of film (shown in Figure 3). In a single model, $S, I$, and $D$ stand for the source, quantum island, and drain electrode, respectively. The applied voltage between $S$ and $D$ is $V_{D S}$, and electrons will transfer in metal island capacitor. If the quantum island can accommodate $N$ electronics and in the existing $N-1$, when the $N$ electrons can be completed metalquantum island-metal tunnel, $V_{D S}$ must meet the following conditions according to the theory of Coulomb blockade [1013]

$$
V_{D S} \geq \min \left(\frac{(N-1 / 2) e}{C_{S}} ; \frac{(N-1 / 2) e}{C_{D}}\right) .
$$

In Formula (1), $e$ represents the electric quantity of a single electron, and $C_{S}$ and $C_{D}$ are the capacitances of source electrode and drain electrode, respectively.

The radius of metal island is $r$, the width between metal island and electrode is $l$, the thickness of films is $D$, the applied voltage on the both ends of each unit is $V_{D S}$, and the transverse and longitudinal number of two-dimensional films are $m$ and $n$, respectively. Based on the scattering theory, the electric current between any two metal islands on the surface of films is $I[14,15]$, which can be estimated as follows:

$$
I=N \cdot e=e D v \int \sigma(\Omega) d \Omega
$$

In Formula (2), $N$ is the number of scattered particles during unit time and in unit scattering angle $d \Omega$ in a certain direction $\Omega$, and $e$ is the electric quantity of a single electron, and $v$ is the speed of electronic transmission. $\sigma(\Omega)$ is the scattering cross-section in $\Omega$ direction.

According to $T$ matrix theory,

$$
\frac{d \sigma_{S D}}{d \Omega}=\frac{4 \pi}{\hbar v}\left|T_{S D}\right|^{2} \rho_{D}\left(E_{F}\right)
$$

in which $\sigma_{S D}$ is the scattering cross-section from source electrode to drain electrode, and $\hbar$ is Planck constant; 


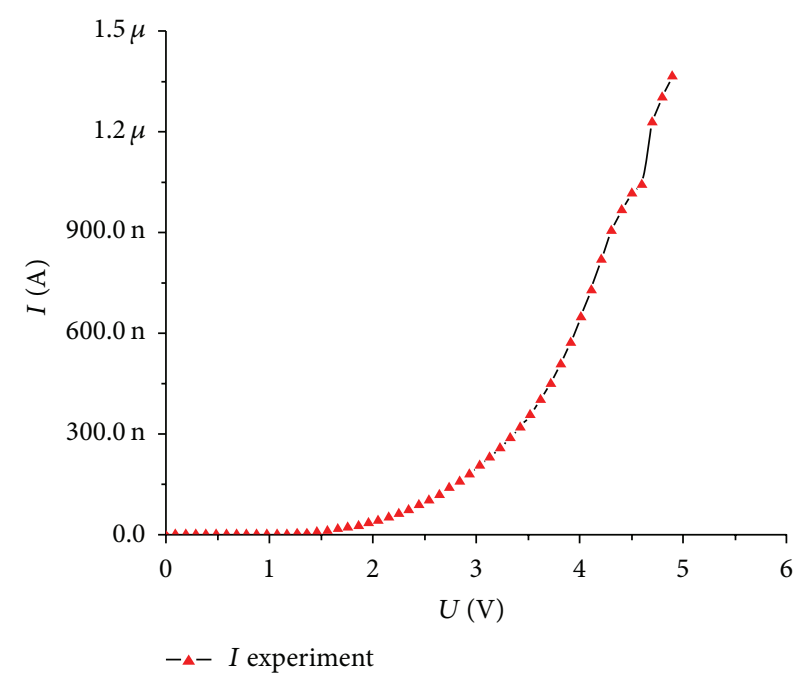

(a)

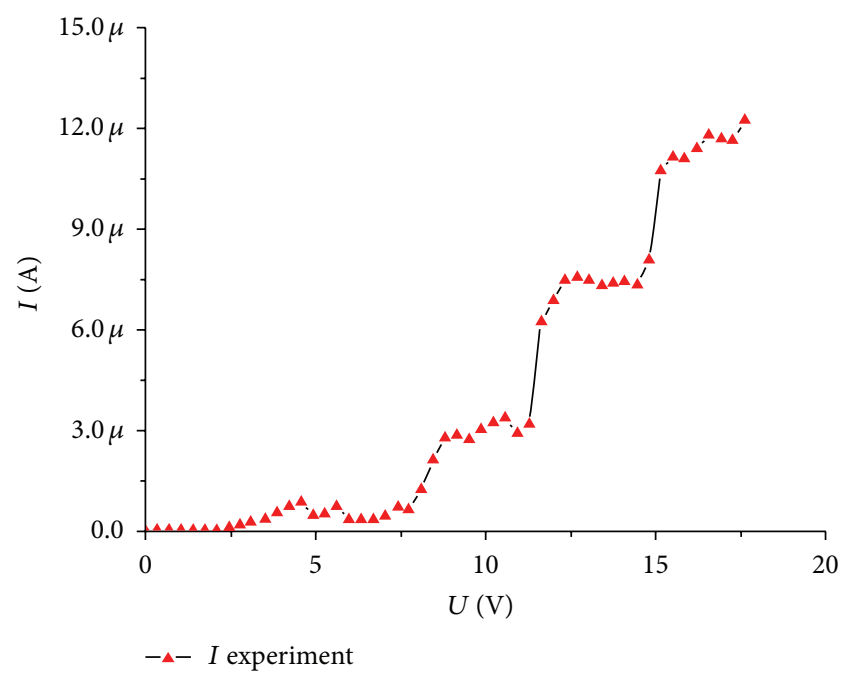

(c)

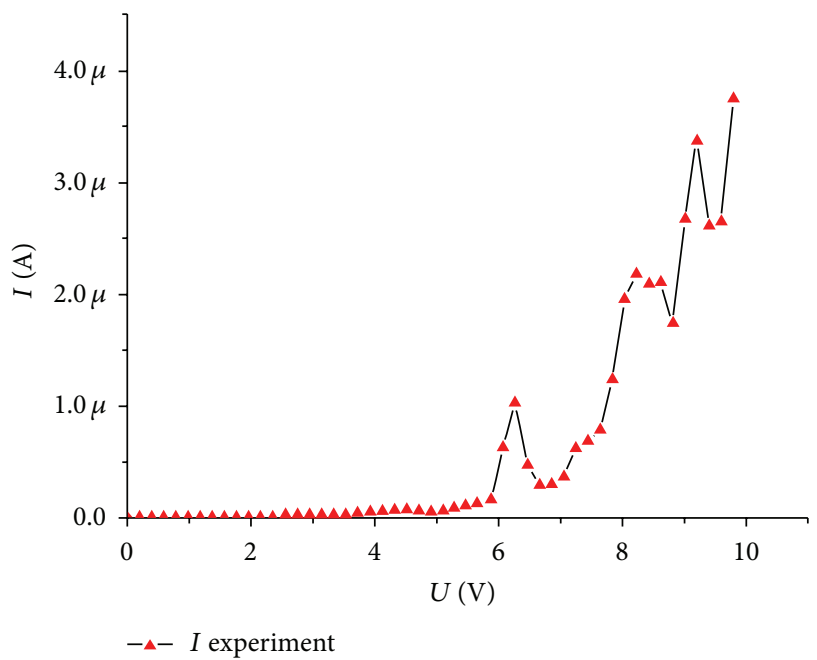

(b)

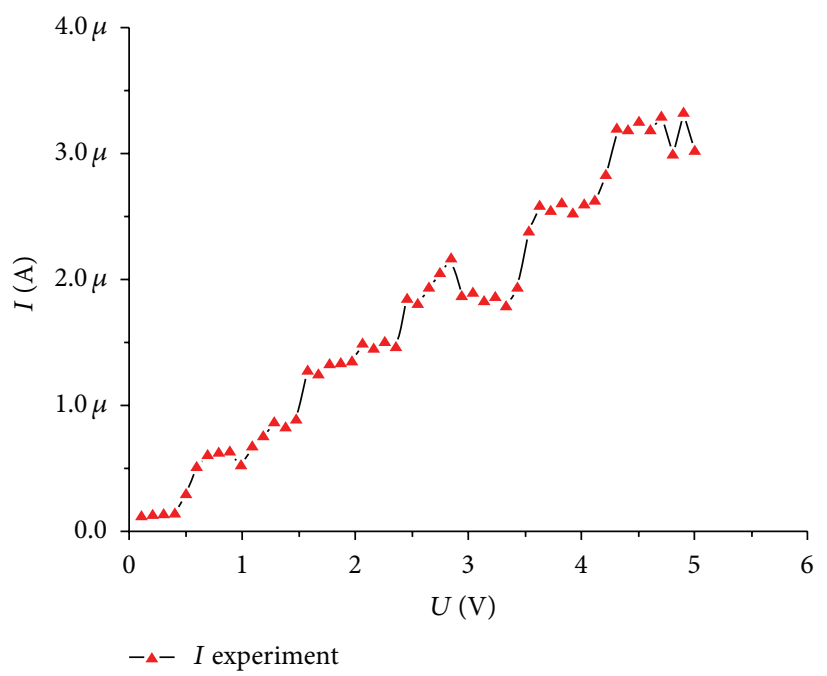

(d)

Figure 1: Volt-ampere characteristics curve of samples with different thickness (20 nm (a), $13 \mathrm{~nm}(\mathrm{~b}), 9 \mathrm{~nm}(\mathrm{c})$, and $4 \mathrm{~nm}$ (d)).

$\rho_{D}\left(E_{F}\right)$ is the density of electronic state of Fermi level. After dragging Formula (3) in Formula (2), we can get the following formulas:

$$
\begin{aligned}
I= & \frac{4 \pi e D}{\hbar}\left|T_{S D}\right|^{2} \int n(E) d E, \\
n(E)= & f(E)\left[1-f\left(E-E_{C}+\Delta E_{S-I}\right)\right] \\
& -f\left(E+\Delta E_{I-D}\right)\left[1-f\left(E-E_{C}\right)\right],
\end{aligned}
$$

where $f(x)=1 /\left(1+e^{\left(x-E_{F}\right) / K_{B} T}\right)$, and in the formulas, $K_{B}$ is Boltzman constant, and the first part on the right of the equal sign of Formula (5) indicates that electrons are scattered from source electrode to quantum island, and the second part suggests that electrons are scattered from quantum island to drain electrode. Using WKB method, we can further get the following formula:

$$
\left|T_{S D}\right|^{2} \sim e^{-\beta l}, \quad \beta=\frac{2 \sqrt{2 \mu E_{0}}}{\hbar},
$$

where $\mu$ is the effective mass of transmission electron and $E_{0}$ is effective barrier height among source electrode-quantumdrain electrode. In an ideal condition, $C_{S}$ is equal to $C_{D}$, so we can obtain that the energy level separation between source electrode and quantum island is equal to the energy level separation between quantum island and drain electrode:

$$
\Delta E_{S \rightarrow I}=E_{C}-e\left(\frac{C_{D}}{C}\right) V_{D S}=\Delta E_{I \rightarrow D},
$$

where $E_{C}=e^{2} / 2 C$, and in the formula, $E_{C}$ is the energy of an electron which transfers from source electrode to drain electrode and goes through quantum island, and $C$ is the total 


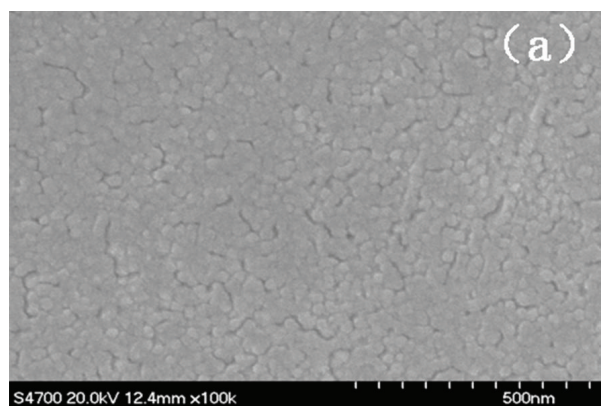

(a)

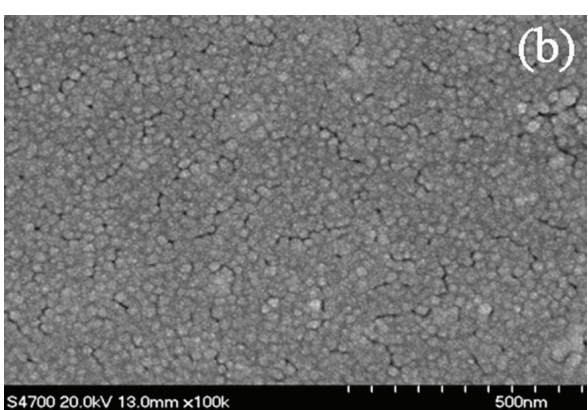

(b)

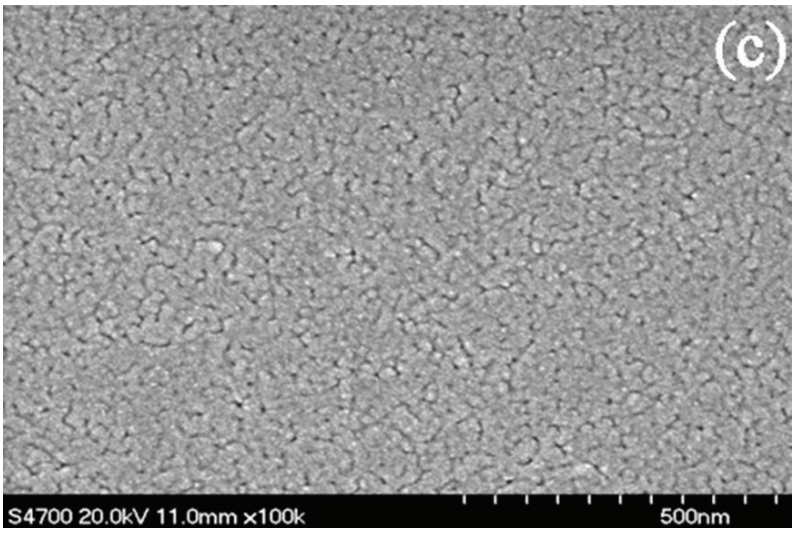

(c)

FIGURE 2: FE-SEM micrographs of Au film with different thickness ((a) $13 \mathrm{~nm}$, (b) $9 \mathrm{~nm}$, and (c) $4 \mathrm{~nm}$ ).

Quantum island

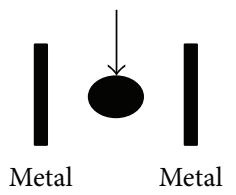

Figure 3: Metal island capacitor model.

capacitance of metallic capacitor. So we can further get the following formula:

$$
\begin{aligned}
I= & \frac{4 \pi e D}{\hbar} \cdot e^{-\left(2 \sqrt{2 \mu E_{0}} / \hbar\right) l} \\
& \times\left[\frac{E_{C}+\Delta E}{1-e^{\left(E_{C}+\Delta E\right) / K_{B} T}}-\frac{E_{C}-\Delta E}{1-e^{\left(E_{C}-\Delta E\right) / K_{B} T}}\right], \\
C_{S}= & C_{D}=\frac{\varepsilon_{0} S}{d}=\frac{2 \varepsilon_{0} r D}{d} .
\end{aligned}
$$

If we supposed the applied total voltage at the surface of film was $V$,

$$
V=m \cdot V_{S D} \cdot \frac{l_{0}}{l}
$$

in which $l_{0}$ is the average width of interspace. It can be known that $l$ is less than $5 \mathrm{~nm}$ from FE-SEM micrographs. So we would obtain the relationship between electric current and voltage under a bias voltage. We dragged the specific parameters in the formula to carry on a calculation, and the calculation results were compared with the experimental results as shown in Figures 4(a) and 4(b).

\subsection{Discussion and Analysis about the Electrical Conductivity} of Films. By comparing Figures 1(a)-1(d), it is clear that, at room temperature, electrical conductivity transfers into nonlinearity from linearity and became step phenomenon with the gradual decrease of films' thickness with the range of current of 0 to $1.5 \times 10^{-5} \mathrm{~A}$. In the experiment, nonlinearity phenomenon can be observed only when the thickness of films dropped to below $20 \mathrm{~nm}$, and step phenomenon can be observed below $10 \mathrm{~nm}$. Under the temperature of $0.9 \mathrm{~K}$, the systematic capacitance $C$ would be less than $10^{-15} \mathrm{~F}$ order of magnitude, and the charging energy $\left(E_{C}=e^{2} / 2 C\right)$ of a single electron would exceed the energy $\left(K_{B} T=77.625 \mu \mathrm{eV}\right)$ of electron's thermal motion, which showed that thermal motion was covered up in low atmospheric pressure. In this way, the phenomenon of electron tunneling can be observed. At room temperature $(T=300 \mathrm{~K}), K_{B} T=25.875 \mathrm{MeV}$, only if the charging energy of a single electron had exceeded this energy, we could observe electron tunneling phenomenon. In this case, the capacitance needed to meet the condition $\left(C \leq 3.1 \times 10^{-18} \mathrm{~F}\right)$, and the total capacitance reduced to $1 / 300$ of the one in low temperature. So, we would observe the phenomenon at $0.9 \mathrm{~K}$, only when the craft size of capacitor was less than 1 micron. And we did the experiment at room 


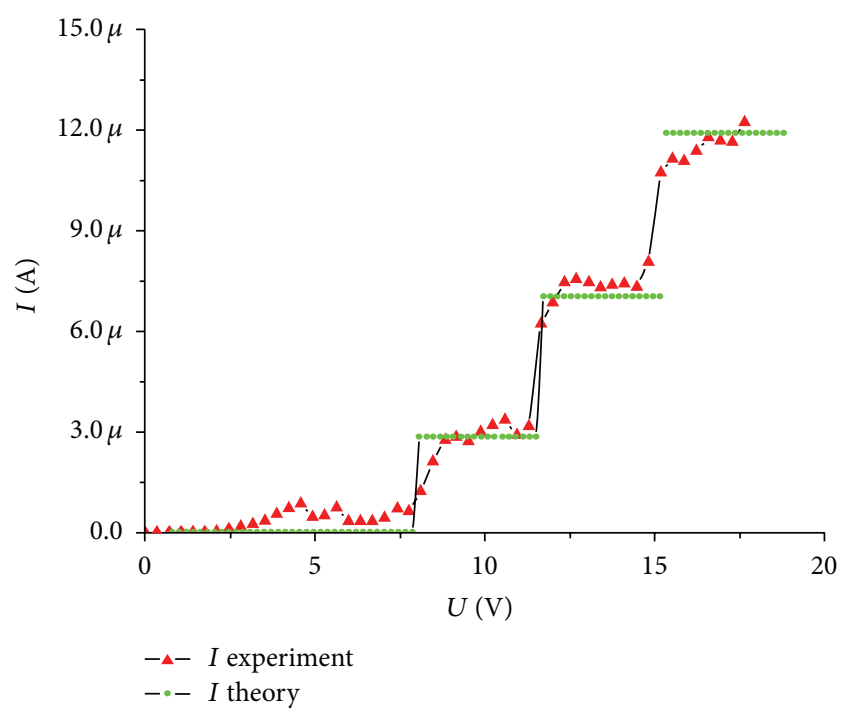

(a) Au film with the thickness of $9 \mathrm{~nm}$

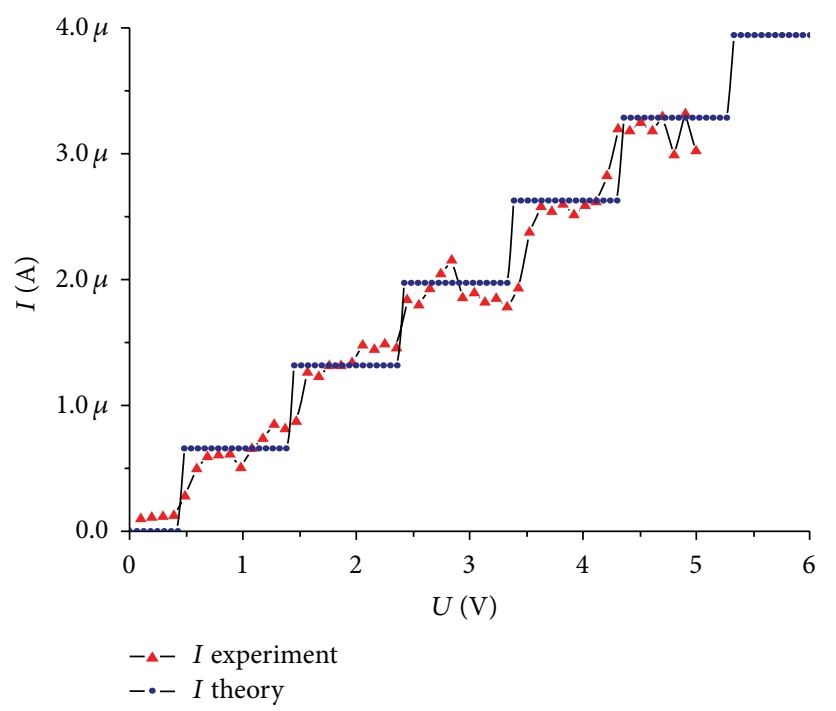

(b) Au film with the thickness of $4 \mathrm{~nm}$

FIgURE 4: Comparing simulation $I-V$ curves with experimental results.

temperature which meant that we could only reduce the size of the capacitor in order to observe electron tunneling phenomenon. So we needed to reduce the thickness of conductive films, and only when the thickness of films was controlled less than 20 nanometers, we could observe the electron tunneling phenomenon.

From FE-SEM micrographs, it could be known that the films were discontinuous, and the particle size was uniform distribution. When electrons transferred at the surface of films, electrons would be scattered in interspace, and there would be Coulomb blockade effect. Figure 4(a) was in comparison with Figure 4(b), and we could find that when the thickness was below $10 \mathrm{~nm}$, the experimental results were close to the calculation ones. Coulomb blockade effect caused by the contact-potential barrier in interspace can be the origin of the nonlinearity and step phenomena of the electrical conductivity of film conductor.

\section{Conclusion}

At room temperature, when the thickness of Au films with discontinuous surface structure was below 20 nanometers, nonlinearity of resistivity can be observed. When the thickness was below 10 nanometers, step phenomena can be obviously observed. The working current was in the range from 0 to $1.5 \times 10^{-5} \mathrm{~A}$. The computational electrical conductivity of films based on the Coulomb blockade theory and scattering theory was consistent well with experimental result. The results showed that the nonlinear electrical conductivity of films conductor was caused by Coulomb blockade effect when electrons transferred in interspaces.

\section{Conflict of Interests}

The authors declare that there is no conflict of interests regarding the publication of this paper.

\section{Acknowledgments}

This work was supported by the 973 Program (Grant no. 2014CB932103), the 863 Program (Grant no. 2013AA032501), the National Natural Science Foundation of China (NSFC), the Scientific Fund from Beijing Municipal Commission of Education (20111001002), the Fundamental Research Funds for the Central Universities, the 111 Project (Grant B07004), and Program for Changiiang Scholars and the Innovative Research Team in University (PCSIRT: IRT1205).

\section{References}

[1] W. Chen, H. Ahmed, and K. Nakazoto, "Coulomb blockade at $77 \mathrm{~K}$ in nanoscale metallic islands in a lateral nanostructure," Applied Physics Letters, vol. 66, no. 24, pp. 3383-3384, 1995.

[2] M. Aslam, I. S. Mulla, and K. Vijayamohanan, "Insulatormetal transition in Coulomb blockade nanostructures," Applied Physics Letters, vol. 79, no. 5, pp. 689-691, 2001.

[3] X. K. Zhao and J. H. Fendler, "Size quantization in semiconductor particulate films," Journal of Physical Chemistry, vol. 95, no. 9, pp. 3716-3723, 1991.

[4] G. R. Wang, L. Wang, Q. Rendeng, J. Wang, J. Luo, and C. Zhong, "Correlation between nanostructural parameters and conductivity properties for molecularly-mediated thin film assemblies of gold nanoparticles," Journal of Materials Chemistry, vol. 17, no. 5, pp. 457-462, 2007.

[5] D. V. Averin and Y. V. Nazarov, "Virtual electron diffusion during quantum tunneling of the electric charge," Physical Review Letters, vol. 65, no. 19, pp. 2446-2449, 1990.

[6] L. J. Geerligs, D. V. Averin, and J. E. Mooij, "Observation of macroscopic quantum tunneling through the Coulomb energy barrier," Physical Review Letters, vol. 65, no. 24, pp. 3037-3040, 1990.

[7] A. T. Tilke, F. C. Simmel, R. H. Blick, H. Lorenz, and J. P. Kotthaus, "Coulomb blockade in silicon nanostructures," Progress in Quantum Electronics, vol. 25, no. 3, pp. 97-138, 2001. 
[8] D. V. Talapin, J. Lee, M. V. Kovalenko, and E. V. Shevchenko, "Prospects of colloidal nanocrystals for electronic and optoelectronic applications," Chemical Reviews, vol. 110, no. 1, pp. 389458, 2010.

[9] C. J. B. Ford, T. J. Thornton, R. Newbury et al., "Transport in GaAs heterojunction ring structures," Superlattices and Microstructures, vol. 4, no. 4-5, pp. 541-544, 1988.

[10] A. Zabet-Khosousi, P. Trudeau, Y. Suganuma, A. Dhirani, and B. Statt, "Metal to insulator transition in films of molecularly linked gold nanoparticles," Physical Review Letters, vol. 96, no. 15, Article ID 156403, 2006.

[11] T. B. Tran, I. S. Beloborodov, J. Hu, X. M. Lin, T. F. Rosenbaum, and H. M. Jaeger, "Sequential tunneling and inelastic cotunneling in nanoparticle arrays," Physical Review B, vol. 78, no. 7, Article ID 075437, 2008.

[12] X. M. Lin, C. M. Sorensen, and K. J. Klabunde, "Digestive ripening, nanophase segregation and superlattice formation in gold nanocrystal colloids," Journal of Nanoparticle Research, vol. 2, no. 2, pp. 157-164, 2000.

[13] C. P. Collier, R. J. Saykally, J. J. Shiang, S. E. Henrichs, and J. R. Heath, "Reversible tuning of silver quantum dot monolayers through the metal-insulator transition," Science, vol. 277, no. 5334, pp. 1978-1981, 1997.

[14] A. Messiah, Quantum Mechanics, vol. 2, North-Holland, Amsterdam, The Netherlands, 1970.

[15] M. B. Isichenko, "Percolation, statistical topography, and transport in random media," Reviews of Modern Physics, vol. 64, no. 4, pp. 961-1043, 1992. 

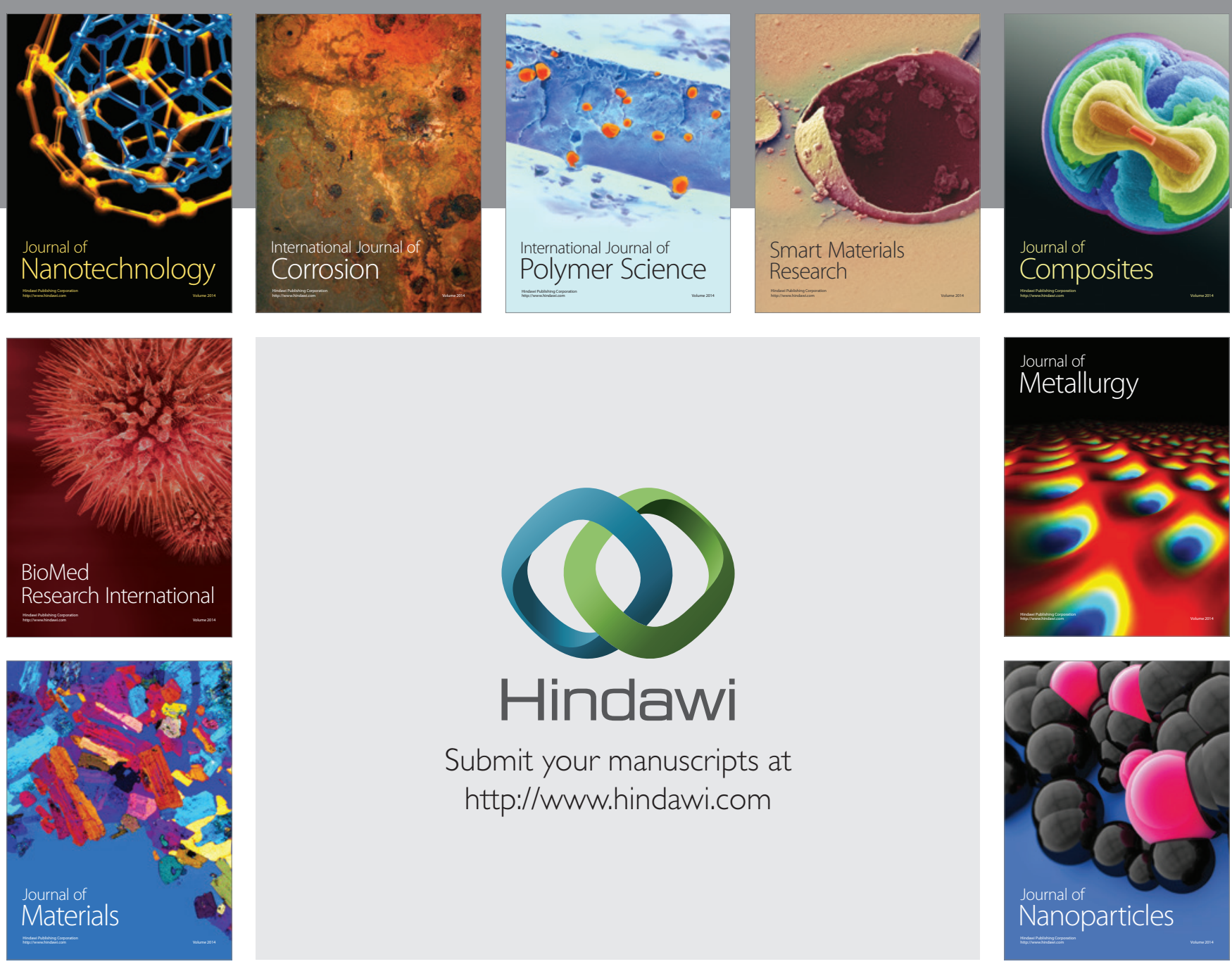

Submit your manuscripts at http://www.hindawi.com
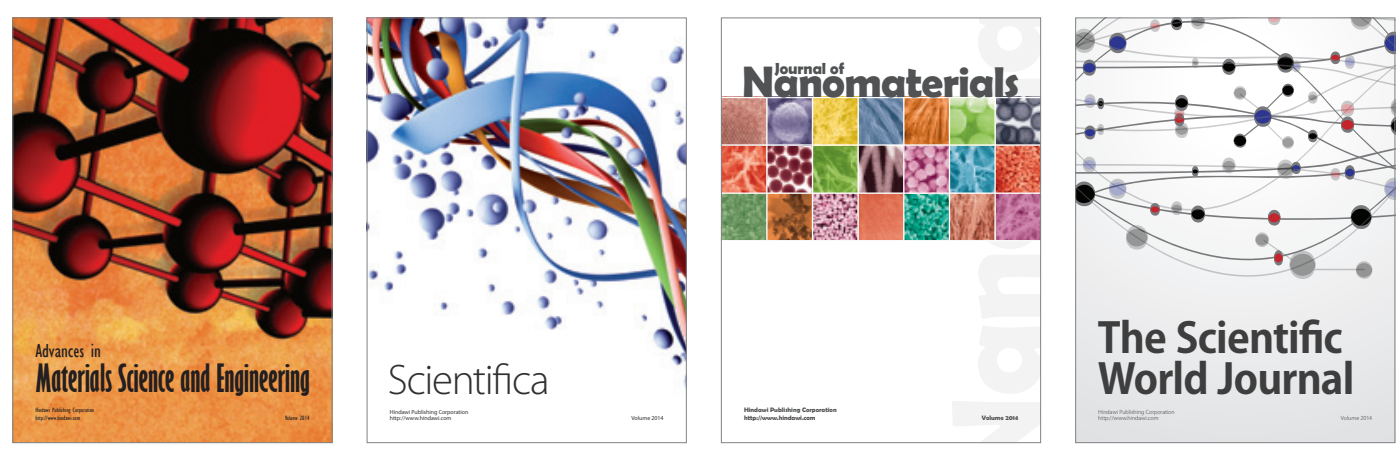

\section{The Scientific World Journal}
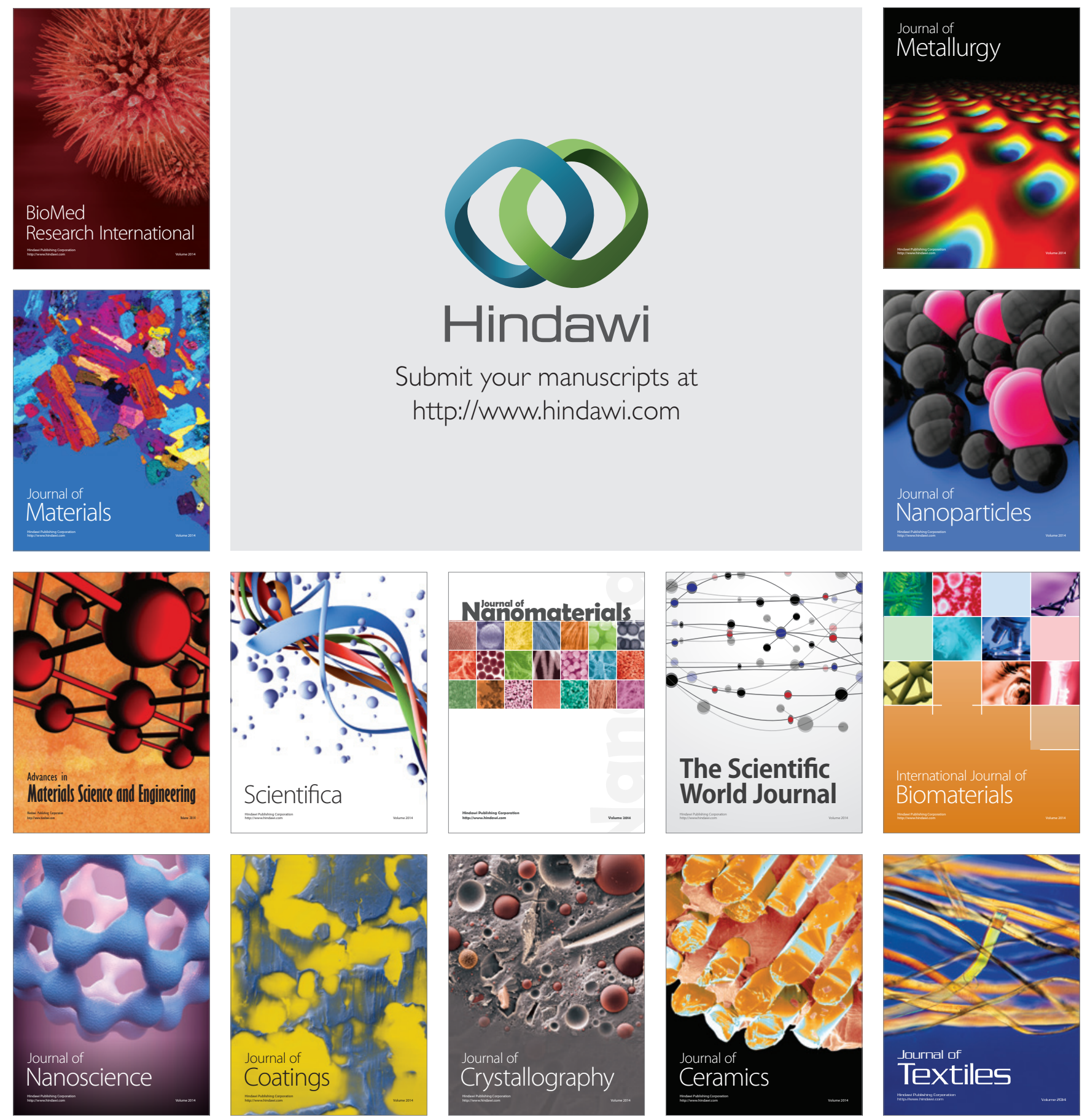